N. E. Piskunov, W. W. Weiss, D. F. Gray, eds.

\title{
Round Table Summary: Radiative Transfer Problems
}

\author{
Nikolai Piskunov \\ Department of Astronomy and Space Physics, Uppsala University \\ Box 515, S-75109 Uppsala, Sweden
}

\begin{abstract}
This Round Table addresses various problems related to the radiative energy transport calculations and the challenges related to the introduction of $3 \mathrm{D}$ hydrodynamic models, models with pulsations, connecting stellar atmospheres to the circumstellar medium etc. We have discussed several examples of environment not properly treated by radiative transfer calculations and outlined future development directions in this field.
\end{abstract}

\section{Introduction}

Radiation carries the energy produced in stellar interiors through the atmosphere into interstellar medium. The ability to understand and properly model radiative transfer (RT) is critical for constructing realistic models of stellar atmospheres, for interpreting observations and deriving accurate stellar parameters (temperatures, masses, abundances etc.) RT calculations involve a whole range of issues. The evaluation of opacity and emissivity intimately relates RT to the chemical composition, molecular and statistical equilibrium of stellar matter, which depends on the local physical conditions (temperature, pressure, magnetic fields etc.), dynamic phenomena (e.g. waves) and radiation field itself. Several assumptions can significantly simplify RT calculations depending on the type of stars and parts of the atmosphere but the assessment of the consequences of such assumptions is not easy.

This round table addresses problems related to the missing physics in the treatment of radiative energy transport in stellar model atmospheres. Specific issues addressed (although not in the same sequence) were: dynamics, 3D, magnetic fields, radiative transport (RT) computing techniques, atomic and molecular data and future directions and challenges. The round table panel consisted of Martin Asplund, Mats Carlsson, Thierry Lanz, Phil Bennett and Nikolai Piskunov. Milan Dimitrijevic, Charles Cowley, Andrew McMurry and Georges Alecian were invited to give oral presentations.

\section{3D models}

The recent success of $3 \mathrm{D}$ hydrodynamic models in matching the observed spectral line profiles without traditional fudge parameters (e.g. Asplund 2003, this volume) clearly indicates directions for the future development of stellar atmo- 
sphere modelling. Radiative energy transport computed consistently with a 3D hydrodynamic model accounts for differences in opacity and emissivity as well as Doppler shifts of various structures on small scales reproducing the average offsets, shapes and strengths of line profiles. The main lesson we have learned is that spectral synthesis for $1 \mathrm{D}$ models is quite different from the average of 3D calculations and thus can never reproduce observations of, for example, cool stars. The development of $3 \mathrm{D}$ radiative transport is not only required by the improvements of theoretical models but also by the new observational evidence for horizontal and vertical chemical and temperature inhomogeneities and the presence of magnetic fields.

Although the first results of the 3D calculations are very encouraging, several effects are still not fully accounted for. One example (Jeff Linsky) is a situation with optically thick spectral lines formed in up- and down-streams in the solar chromosphere with Doppler shifts sufficient to allow the photons to leak out. Such a situation is significant to the overall energy balance as was shown for the 1D case (Mats Carlsson) but is not properly accounted for in the $3 \mathrm{D}$ case due the still limited number of frequency points. In the latest 3D (LTE) calculations by Martin Asplund a strategic choice of wavelengths for the opacity sampling allows one to account for energy transport between the up- and down-flows while computing the emerging spectra, but no self-consistent model can yet be produced.

\section{Dynamics}

In parallel with the understanding that spatially averaged spectra do not necessarily coincide with the calculations for $1 \mathrm{D}$ models we also see that the time average differs significantly from the predictions of static models. Andrew McMurry (this volume) showed the results of RT calculations through the outer parts of the solar atmosphere with acoustic shocks. Lee Anne Willson pointed out that in such models with a single dominating pulsation mode at a certain point the solution reaches a semi-periodic stage significantly different from a static model. The situation may be even more complicated with a superposition of multi-frequency waves and in this case a semi-periodic stage may not even exist.

Jeff Linsky brought up two other examples where a static approximation does not reproduce dynamical properties. One is the thermal bifurcation in the outer atmospheric layers: due to formation of the $\mathrm{CO}$ molecule which is also an efficient cooling agent, rather stable structures with two dramatically different temperatures can coexist at the same distance from the center of a star. The second is the formation of super-waves formed in regions with a rich spectrum of acoustic oscillations. These super-waves have propagation and dissipation properties quite different from any of the original oscillations.

Dynamic phenomena with different time scales result in atmospheric structure remarkably different from a model prediction assuming instant reaction of stellar matter to a change in physical conditions. For example, in the solar case hydrogen ionization cannot keep up with the pressure perturbations (waves). Ignoring this effect would result in an incorrect energy balance as the excess 
kinetic energy will be converted into enhanced ionization which does not occur in reality.

\section{Magnetic fields}

The presence of magnetic fields creates a preferred direction in the atmosphere causing several effects. One of them (important for magnetic Ap stars) is a modification of radiative acceleration due to Zeeman broadening of individual spectral lines (Georges Alecian, this volume). Numerical simulations show that the amplification of radiative acceleration is sensitive to field strength and orientation and, therefore, should lead to a rather complex pattern of chemical diffusion in magnetic Ap stars. Viktor Khalack mentioned that the recent progress in Doppler Imaging and Magnetic Doppler Imaging allows direct verification of the chemical stratification patterns predicted by the models once reliable predictions are made.

The interaction of diffusion mechanisms with dynamic phenomena like pulsations well known for cool Ap stars was discussed by Werner Weiss and Georges Alecian. It seems that the very different characteristic velocities involved (1 $\mathrm{cm} \mathrm{s}^{-1}$ versus $1 \mathrm{~km} \mathrm{~s}^{-1}$ ) prevent any significant interaction.

Several other examples of magnetic fields affecting energy transport in stellar atmospheres have been discussed including enhanced blanketing, reduced convection and the Lorentz force. Thierry Lanz summarized this part of the discussion by saying that magnetic fields destroy 1D geometry of stellar atmospheres and require a 3D treatment of RT. The main limiting factors are the number of different angles and frequency points, and ultimately - the computing power.

\section{Computational techniques and completeness of atomic and molec- ular line lists}

Charles Cowley (this volume) presented a comparison of the line opacity calculations using different frequency grids. Although, the arithmetic mean opacity over a small wavelength interval is totally dominated by the strong lines, the harmonic mean better reflects the opacity of weak lines and, therefore, is strongly affected by incompleteness of the line lists. Opacity sampling with a small number of frequencies is not capable of fully reproducing the mean values. Bengt Gustafsson pointed out that for the total opacity the presence of a smooth continuous component offsets the problem of missing weak lines, while Thierry Lanz described the opacity re-normalization techniques often used in combination with opacity sampling. He also mentioned that the exact errors depend on the physical conditions (temperature and pressure) and wavelength so that individual errors cancel out during model calculations. This does not solve the problem of incomplete line lists which is particularly severe for molecular lines where even strong lines are often not well known.

Milan Dimitrijevic (this volume) presented chemical reactions producing atoms in highly excited states. Such reaction channels can, for example, create important perturbations of the level populations for hydrogen. The first atomic data which allows calculation of this effect were produced in Belgrade. Charles 
Cowley asked about data for spectral line shifts due to collisions with charged particles. Milan Dimitrijevic replied that in the atmospheres of cool star line broadening is mostly produced by collisions with neutral particles keeping line profiles symmetric. At temperatures of $10000 \mathrm{~K}$ or larger collisions with electrons start producing shifts and broadening of spectral lines and creating asymmetric profiles. Some important examples are helium and silicon lines where line position and shape were reproduced by accurate calculations of Stark effect. Parameters for many important transitions are available, for example, from VALD database.

Robert Kurucz reminded that his line lists include Landé factors and, therefore, can be used for computing RT with magnetic fields. He also asked if Milan Dimitrijevic is planing to make available his data for Stark broadening and Stark shifts. Milan said that the work on a database is in progress.

\section{Future directions and challenges}

I like very much the comment of Thierry Lanz on my question about what determines the direction of future development: "Is it what we know or is what we do not know?" Thierry said: "... I think this is a question of what is most important at any given time. When we started to work on NLTE blanketing about 10 years ago there was a big debate about if it was more important to have departures from LTE in the existing models or to have LTE blanketed models. The only way to solve this problem was to compute NLTE line blanketed models. Today I can say that one would probably make a bigger mistake by making a NLTE model without blanketing than an LTE model with blanketing. Now that we have done this, there is no point of continuing doing the same thing for the next 20 years because we know how. We should consider what to do next. Since we know how to compute NLTE line blanketed models with a large number of lines and levels we should try to incorporate it into 3D hydrodynamics in order to better predict e.g. radiative force. This is a direction we should take. My feeling is that 1D fully blanketed models with radiation hydrodynamics are within reach and several people are actually working on such models but the real breakthrough will happen when this will be implemented in 3D."

Acknowledgments. I would like to thank Paul Barklem for excellent audio and video recording of the round table and for his help with the manuscript. 